\title{
Long-chain non-coding RNA GACAT1 promotes development and progression of breast cancer by targeting microRNA-875-3p
}

\author{
QINGHUA WANG $^{1}$, JIE XUE $^{1}$, QINGFANG REN $^{2}$, XIAONA LI $^{1}$ and XIAOLI QIU ${ }^{1}$ \\ Departments of ${ }^{1}$ Breast Surgery and ${ }^{2}$ Medical Oncology, Linyi Cancer Hospital, Linyi, Shandong 276000, P.R. China
}

Received October 25, 2019; Accepted December 20, 2019

DOI: $10.3892 / \mathrm{ol} .2020 .11260$

\begin{abstract}
Role of long-chain non-coding ribonucleic acid (lncRNA) GACAT1 in the development of breast cancer and its possible mechanism were investigated. The levels of GACAT1, microRNA-875-3p and Stonin2 (STON2) in breast cancer tissues and adjacent normal tissues were detected by quantitative real-time polymerase chain reaction (qRT-PCR). The level of GACAT1 in breast cancer cell lines was further explored. The effects of GACAT1 and microRNA-875-3p on cell proliferation and cycle were detected by cell counting kit-8 (CCK-8) and flow cytometry. The binding relationship between microRNA-875-3p and STON2, microRNA-875-3p and GACAT1 was examined by a dual luciferase reporting assay. It was confirmed by rescue experiments whether GACAT1 canregulate the level of STON2 by binding to microRNA-875-3p. GACAT1 level was clearly enhanced in breast cancer tissues compared to that of the adjacent tissues. Similar result was observed in breast cancer cell lines. Upregulation of GACAT1 promoted the proliferation and cycle of breast cancer cells including MCF-7 and BCap-37. The dual luciferase reporting assay results indicated that GACAT1 had a binding relationship with microRNA-875-3p. Further experiments confirmed that microRNA-875-3p was conspicuously downregulated in breast cancer tissues, and upregulation of microRNA-875-3p could inhibit the proliferation ability of MCF-7 and BCap-37 cells, and partially reversed the promoting effect of GACAT1 on cell cycle. Through bioinformatics prediction and dual luciferase reporter gene experiments, we found that STON2 might be a target gene of microRNA-875-3p. Overexpression of STON2 could partially abolish the effect of microRNA-875-3p on cell proliferation and cycle of MCF-7 and BCap-37 cells. GACAT1 can participate in the progression of breast cancer by promoting the proliferation and cycle of breast cancer cells. The mechanism
\end{abstract}

Correspondence to: Dr Xiaoli Qiu, Department of Breast Surgery, Linyi Cancer Hospital, 6 Lingyuan East Street, Linyi, Shandong 276000, P.R. China

E-mail: 278852691@qq.com

Key words: breast cancer, GACAT1, microRNA-875-3p, STON2 may be through the regulation of the level of STON2 by adsorbing microRNA-875-3p.

\section{Introduction}

Breast cancer is a highly heterogeneous malignant tumor, and its morbidity and mortality are second only to lung cancer, which poses a great threat to human health (1). In recent years, the mortality rate of breast cancer has been increasing year by year (2). Although the prognosis of breast cancer patients is greatly improved under comprehensive treatment, $20-25 \%$ of patients will eventually have local recurrence or even distant metastasis, eventually leading to death. Studies have shown that the development, early diagnosis, comprehensive treatment and prognosis of breast cancer are closely related to the abnormal level of non-coding ribonucleic acid (RNA) (3), so it is necessary to explore the pathogenesis of breast cancer from the perspective of non-coding RNA.

Long-chain non-coding RNA (lncRNA) is a RNA molecule that is more than $200 \mathrm{nt}$ in length and has been proved to be involved in various biological processes in cells $(4,5)$. lncRNA can actively participate in the regulation of various biological functions and plays an important role in a variety of tumors mainly through epigenetic changes, cell cycle regulation, regulation of miRNAs and participation in signaling pathways $(6,7)$. For instance, lncRNA plays an important role in the development of breast cancer (8). By gene chip high-throughput analysis of differential level of lncRNA in breast cancer and normal breast tissue, studies have found that 220 lncRNAs are abnormally expressed in breast cancer tissues, of which 129 lncRNAs are specifically expressed in breast cancer and can be used as breast cancer biomarkers (9). Another study found that lncRNA H19 is overexpressed in estrogen receptor positive breast cancer and promotes breast cancer growth and proliferation (10). IncRNA GACAT1 is highly expressed in gastric cancer and closely related to the prognosis of gastric cancer, which can be used as a tumor marker for gastric cancer $(11,12)$. Through previous studies, we found that GACAT1 is also highly expressed in breast cancer, but the study on GACAT1 in breast cancer has not been reported.

MicroRNAs (miRNAs) are a class of small-molecule non-coding RNAs found in eukaryotes. They are composed of $\sim 22$ nucleotides and bind to the corresponding target mRNA, resulting in degradation of target mRNA or inhibited 
post-transcriptional translation. At present, scientists have identified 474 miRNAs in the human genome, which could regulate $\sim 30 \%$ of the encoded protein genes, and each miRNA regulates $\sim 100$ target mRNAs (13). The relationship between miRNAs and human life activities and diseases is receiving increasing attention. In particular, recent studies have shown that they are closely related to the occurrence and development of human diseases. For example, in patients with lung cancer, the level of miRNAlet-7 is conspicuously reduced. Meanwhile, let-7a transfection into A549 cell line could inhibit lung cancer cell proliferation (14). In the study on chronic lymphocytic leukemia, it was found that the level of miR-15 and miR-16 in leukemia cells of patients with chronic lymphocytic leukemia is downregulated, while the level of B-cell lymphoma-2 (Bcl-2) is increased. There is a significant negative correlation between miR-15 and miR-16 expression and Bcl-2 protein levels, and miR-15 and miR-16 functions by targeting degradation of Bcl-2 (15).

In the present, through bioinformatics analysis, it was found that microRNA-875-3p might be a potential target gene for long-chain non-coding RNA GACAT1, moreover, its expression was low in breast cancer tissues and cells. This study investigated the role of GACAT1 in breast cancer to verify whether it could function by degrading microRNA-875-3p.

\section{Patients and methods}

General information. Fresh breast cancer tissue and adjacent normal tissues were collected from 24 patients with breast cancer who underwent surgery. The enrollment conditions included: i) exclusion of other malignant tumor history; ii) first surgery, no radiotherapy before surgery; iii) the pathology diagnosis was performed by the pathologist's reading film; iv) the clinical medical record data and the postoperative follow-up data were complete. This study was approved by the Ethics Committee of Linyi Cancer Hospital (Linyi, China). Signed informed consents were obtained from all participants before the study. The specimens were taken from the tumors removed during the operation, and stored in a liquid nitrogen tank within 15 min.

Cell culture. Normal Hs578Bst cells as well as breast cancer cell lines MDA-MB-231, BCap-37 and MCF-7 were purchased from American Type Culture Collection (ATCC). The cells were cultured in Dulbecco's modified Eagle's medium (DMEM) (Gibco; Thermo Fisher Scientific, Inc.) (containing 10\% fetal bovine serum (FBS), $100 \mathrm{U} / \mathrm{ml}$ penicillin and $0.1 \mathrm{mg} / \mathrm{ml}$ streptomycin) at $37^{\circ} \mathrm{C}$, in a $5 \% \mathrm{CO}_{2}$ incubator.

Cell transfection. The cells were inoculated one day before the transfection. The next day, plasmid, miRNA mimics and their respective negative control sequences (50-100 nM) were transfected by Lipofectamine 3000 (Invitrogen; Thermo Fisher Scientific, Inc.). RNase-free diethyl pyrocarbonate (DEPC) water (both from Beyotime) and Eppendorf (EP) tubes were used for transfection.

Tissue and cell total RNA extraction and quantitative real-time polymerase chain reaction ( $q R T-P C R)$. Extraction was carried out by the TRIzol method (Invitrogen; Thermo Fisher Scientific,
Inc.). Chloroform $(200 \mu \mathrm{l})$ was added to each $1 \mathrm{ml}$ of TRIzol-dissolved EP tube, mixed by inversion, placed in an ice box for $5 \mathrm{~min}$, and centrifuged at $4^{\circ} \mathrm{C}, 12,000 \mathrm{x} \mathrm{g}$ for $15 \mathrm{~min}$ carefully pipeting. The extracted RNA was applied to the NanoDrop 2000c assay to determine its concentration and the $260 / 280$ absorbance was used to evaluate the quality. The measurement was repeated twice, and the 260/280 absorbance ratio was between 1.8 and 2.0. The total RNA was then reverse-transcribed into complementary deoxyribonucleic acid (cDNA) which was used for further real-time PCR. Detection of the level of the target gene was performed using an ABI StepOnePlus real-time quantitative fluorescent PCR instrument by the SYBR real-time qPCR kit (Applied Biosystems; Thermo Fisher Scientific, Inc.). Each well contained $10 \mu \mathrm{l}$ SYBR-Green Master Mix, $1 \mu 1$ upstream primer, $1 \mu$ l downstream primer, $0.4 \mu \mathrm{l}$ 50X Rox Dye, $2 \mu \mathrm{l}$ cDNA and $5.6 \mu 1$ enzyme-free water. The reaction conditions were $95^{\circ} \mathrm{C}$ for $10 \mathrm{~min}, 95^{\circ} \mathrm{C}$ for $15 \mathrm{sec}$ and $60^{\circ} \mathrm{C}$ for $60 \mathrm{sec}$, for 40 cycles. The data were statistically analyzed using the StepOne Software version v2.1 (Applied Biosystems; Thermo Fisher Scientific, Inc.) software and the primer sequences were derived as follows: microRNA-875-3p, forward, 5'-ACACTCCAGCTGGGUAUA CCUCAGUUUUAU-3' and reverse, 5'-CTCAACTGGTGTC GTGGAGTCGGCAATTCAGTTGAGCACCUGA-3'; U6, forward, 5'-CTCGCTTCGGCAGCAGCACATATA-3' and reverse, 5'-AAATATGGAACGCTTCACGA-3'; GACAT1, forward, 5'-ACCGGAGGAAAATCCCTAGC-3' and reverse, 5'-CCATAAAAGGGGCGGCTG-3'; GAPDH, forward, 5'-GAAGAGAGAGACCCTCACGCTG-3' and reverse, 5'-ACTGTGAGGAGGGGAGATTCAGT-3'; Stonin2 (STON2), forward, 5'-ACCATGTGATTGCCACCCAC and reverse, 5'-AGCTCTCGGACTGGTCTGG-3'.

Luciferase reporting assay. Reporter plasmid was constructed and termed pmirGL0-STON2-7 7 UTR-wild type (Wt) or pmirGLO-STON2-37 UTR-mutant (Mut), which were inserted into the dual-fluorescence of wild-type and mutant STON2-3'UTR, respectively. Breast cancer cells were seeded into 48-well plates, and the plasmid was co-transfected with miRNA-875-5p mimics, then the luciferase activity was detected $48 \mathrm{~h}$ later.

Detection of cell cycle. Twenty-four hours after transfection, cells were collected, washed twice with phosphate-buffered saline (PBS) and then $1 \mathrm{ml}$ of DNA Staining solution (Takara) was added, vortexed and mixed, and incubated at room temperature in the dark. After $30 \mathrm{~min}$, the samples were detected by MACS flow cytometry (Beckman Coulter).

Cell proliferation. Transfected cells were seeded into 96-well plates (Corning, Inc.) at a density of $3-5 \times 10^{3}$ cells per well, and 10 replicate wells were set for each group. $100 \mu \mathrm{l}$ of a mixture of cell counting kit-8 (CCK-8) reagent (Dojindo) premixed in a ratio of 1:10 was added and then the cells were incubated in a $37^{\circ} \mathrm{C}$ incubator for $1 \mathrm{~h}$. Then the enzyme was added. The optical density (OD) value of each replicate well was measured by microplate reader at $450 \mathrm{~nm}$ wavelength.

Statistical analysis. The measurement data were expressed as mean \pm standard deviation, and t-test was used to examine the 
A

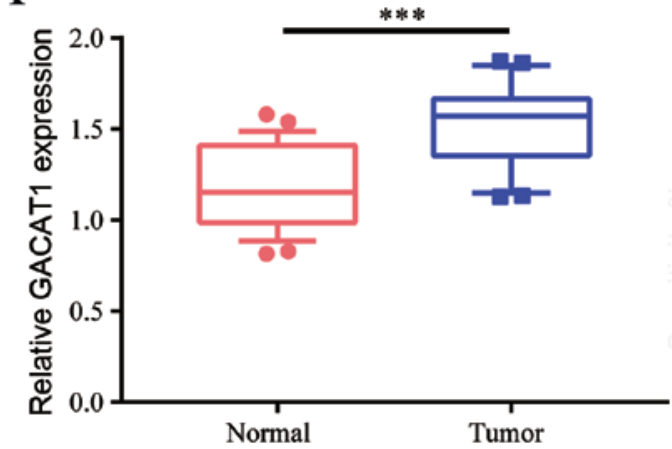

$\mathrm{C}$

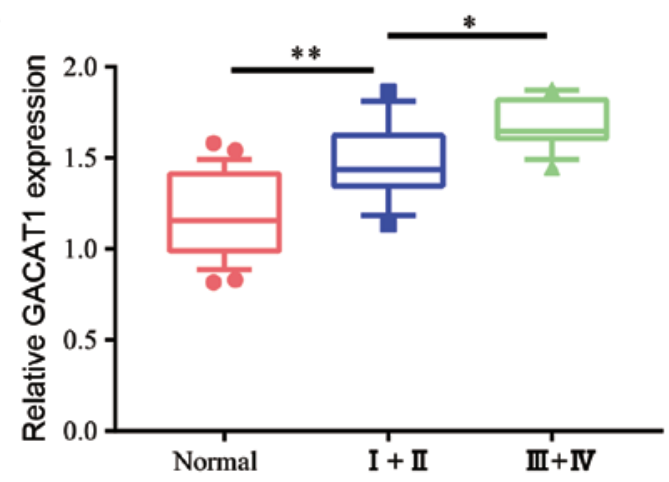

B

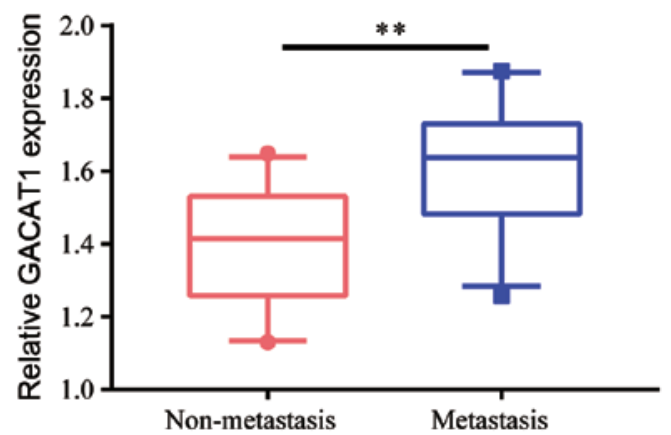

D ROC curve: ROC of Data 4

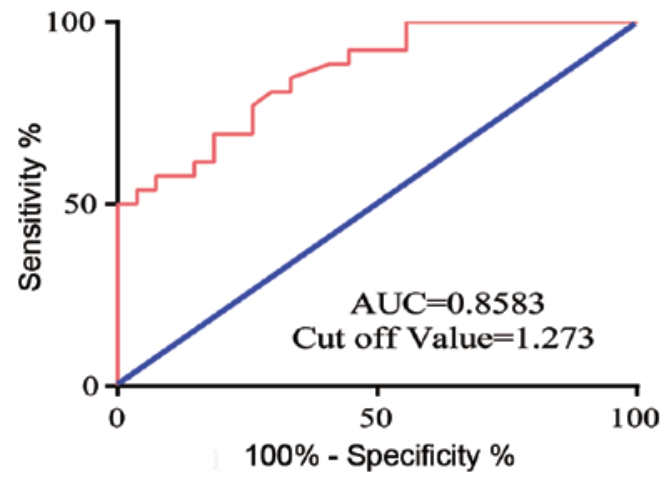

Figure 1. GACAT1 is significantly highly expressed in breast cancer. (A) Compared with adjacent tissues, GACAT1 is highly expressed in breast cancer tissues. (B) GACAT1 expression is significantly higher in patients with cancer metastasis than in non-metastatic group. (C) GACAT1 expression is significantly higher in patients with tumor stage III to IV than those with stage I to II. (D) Survival curve analysis showed that GACAT1 can be used as a diagnostic marker, AUC $=0.8583$, cut off value $=1.273 .{ }^{*} \mathrm{P}<0.05,{ }^{* *} \mathrm{P}<0.01$ and ${ }^{* * *} \mathrm{P}<0.001$.

mean difference between two groups. Single factor variance analysis was used when multiple sets of data meet normality and the homogeneity of the variance. Pairwise comparison was used if there was a difference between the groups. Nonparametric test was used when the data did not meet normality and the homogeneity of the variance. $\mathrm{P}<0.05$ was considered statistically significant.

\section{Results}

GACAT1 is clearly expressed in breast cancer. First, we examined the level of GACAT1 in 24 breast cancer patients. The results showed that microRNA-875-3p was conspicuously expressed in breast cancer tissues compared to that of adjacent tissues (Fig. 1A). Furthermore, we found that in patients with cancer metastasis, the level of GACAT1 was conspicuously higher than that of patients without metastasis (Fig. 1B). We then examined the level of GACAT1 in breast cancer at different stages, and the results showed that GACAT1 level was conspicuously higher in patients with grade III to IV breast cancer than in patients with stage I to II (Fig. 1C). By analyzing the clinical information of breast cancer patients, the survival curve was plotted and the results showed that the area under the curve was 0.8583 and the cutoff value was 1.273 (Fig. 1D), which indicated that GACAT1 can be used as a biomarker for breast cancer.

GACAT1 promotes proliferation and cycle of breast cancer cells. In breast cancer cell lines, we also examined the
GACAT1 level. The results showed that it was generally highly expressed in breast cancer cell lines compared to normal breast cells (Fig. 2A). After transfection of the GACAT1 overexpression plasmid in MCF-7 and BCap-37 cells, we examined the efficiency of transfection, and the results showed that GACAT1 was high and stably expressed (Fig. 2B). Subsequently, we applied CCK-8 assay and flow cytometry to examine the effect of GACAT1 on proliferation and cell cycle of breast cancer cells. The results showed that the upregulation of GACAT1 conspicuously increased the proliferation of BCap-37, and also clearly promoted the cell cycle of BCap-37 (Fig. 2C and D), which was consistent with MCF-7 cells (Fig. 2E and F). These results demonstrated that GACAT1 promoted proliferation and cell cycle of breast cancer cells.

GACAT1 works by adsorbing microRNA-875-3p. Through bioinformatics analysis, we found that GACAT1 might bind to microRNA-875-3p, and confirmed their binding by dual luciferase reporter gene experiments (Fig. 3A). Further studies found that microRNA-875-3p was conspicuously under expressed in breast cancer tissues (Fig. 3B). After upregulating the level of GACAT1 in BCap-37 and MCF-7 cells, the level of microRNA-875-3p was clearly decreased (Fig. 3C). The above results indicated that GACAT1 can inhibit the level of microRNA-875-3p. To verify whether GACAT1 functioned by targeting microRNA-875-3p, we first upregulated GACAT1 and then overexpressed microRNA-875-3p as well, and found that the effect of GACAT1 on cell proliferation in BCap-379 
A

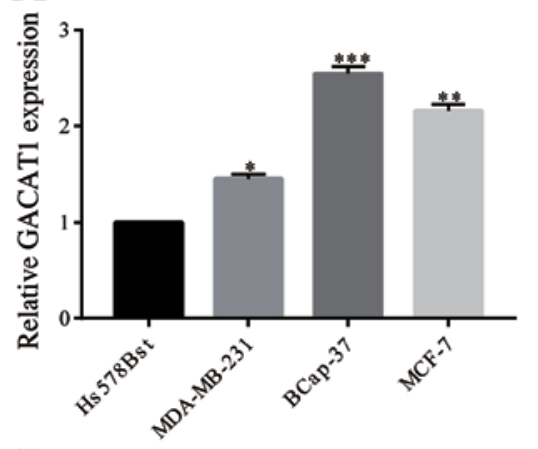

B

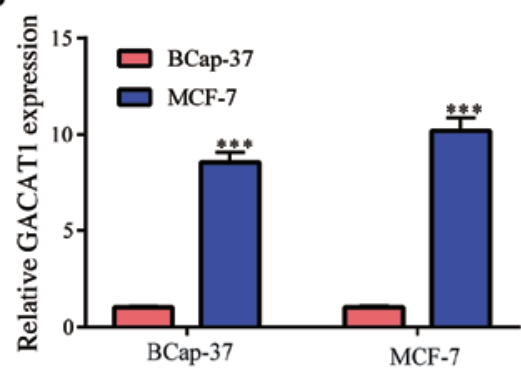

C

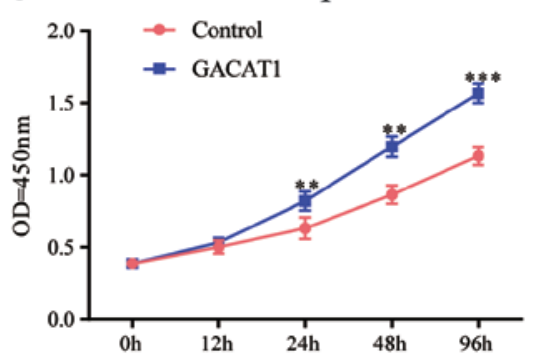

E

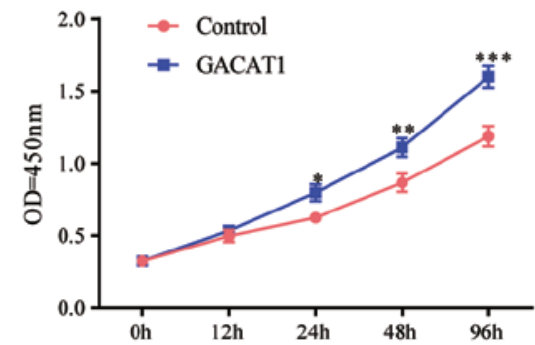

D

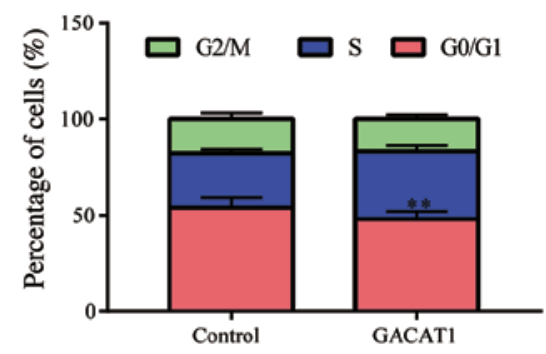

F

MCF-7

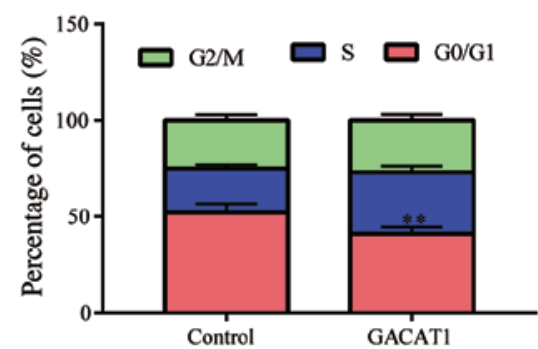

Figure 2. GACAT1 promotes proliferation and cycle of breast cancer cells. (A) GASAT1 expression is generally increased in breast cancer cells. (B) GACAT1 can be stably expressed after transfection of GACAT1 overexpression plasmid in MCF-7 and BCap-37 cells. (C and D) Cell proliferation and cycle can be promoted after GACAT1 is upregulated in BCap-37 cells. (E and F) In MCF-7 cells, upregulation of GACAT1 can significantly promote cell cycle and cell proliferation. ${ }^{*} \mathrm{P}<0.05,{ }^{* *} \mathrm{P}<0.01$ and ${ }^{* * *} \mathrm{P}<0.001$.

A

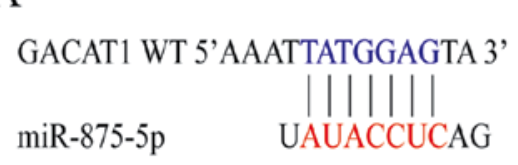

GACAT1 MUT 5'AAAATACCTCTA 3'

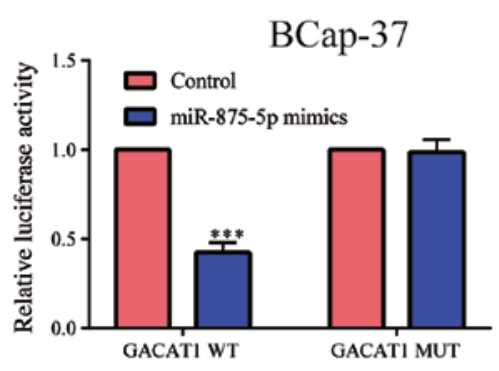

MCF-7

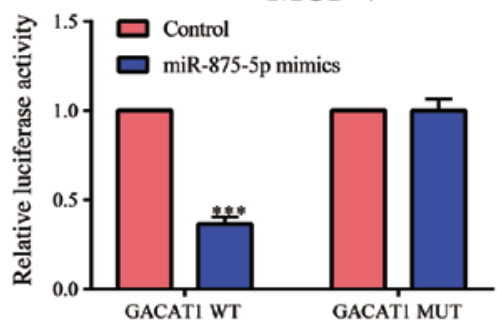

B

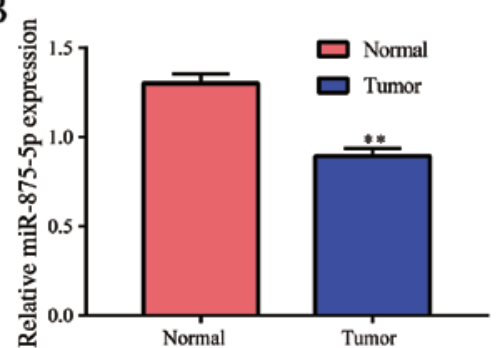

$\mathrm{D}$
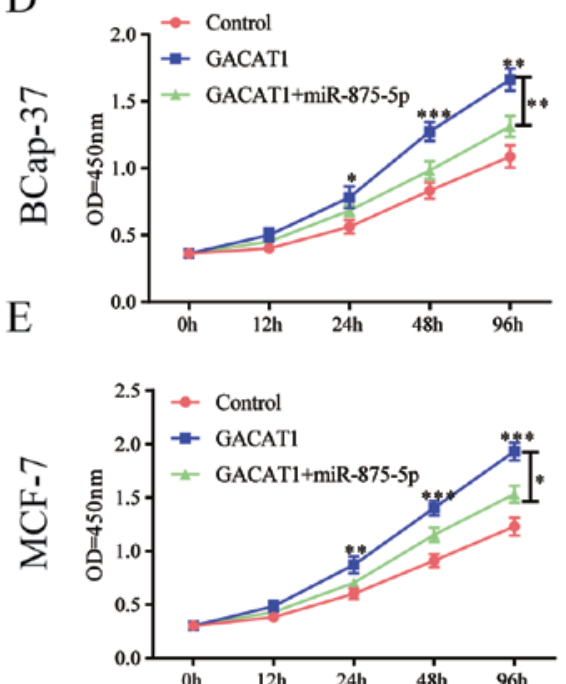

C
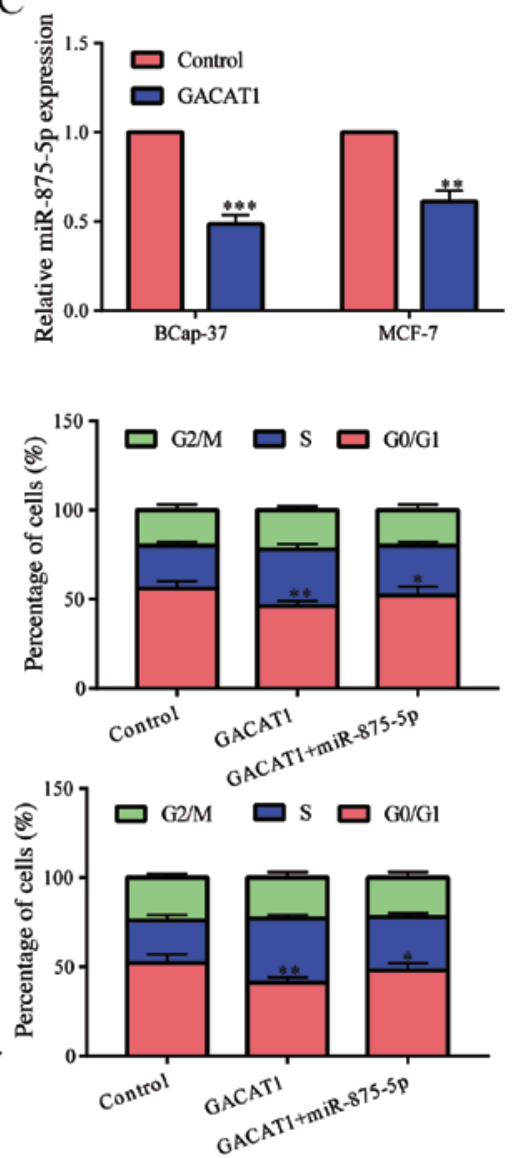

Figure 3. GACAT1 works by adsorbing miR-875-5p. (A) Dual luciferase reporting assay results show that GACAT1 has a binding relationship with miR-875-5p. (B) miR-875-5p is significantly underexpressed in breast cancer cells. (C) After GACAT1 is upregulated in MCF-7 and BCap-37 cells, the expression of miR-875-5p was significantly decreased. (D and E) Upregulation of miR-875-5p in MCF-7 and BCap-37 cells partially reversed the promoting effect of GACAT1 on cell proliferation and cell cycle. ${ }^{*} \mathrm{P}<0.05,{ }^{* *} \mathrm{P}<0.01$ and ${ }^{* * *} \mathrm{P}<0.001$. 
A

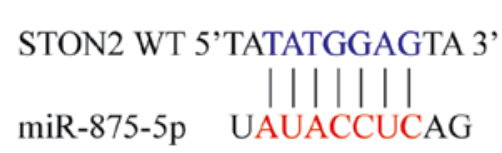

STON2 MUT 5'TAAUACCTCTA
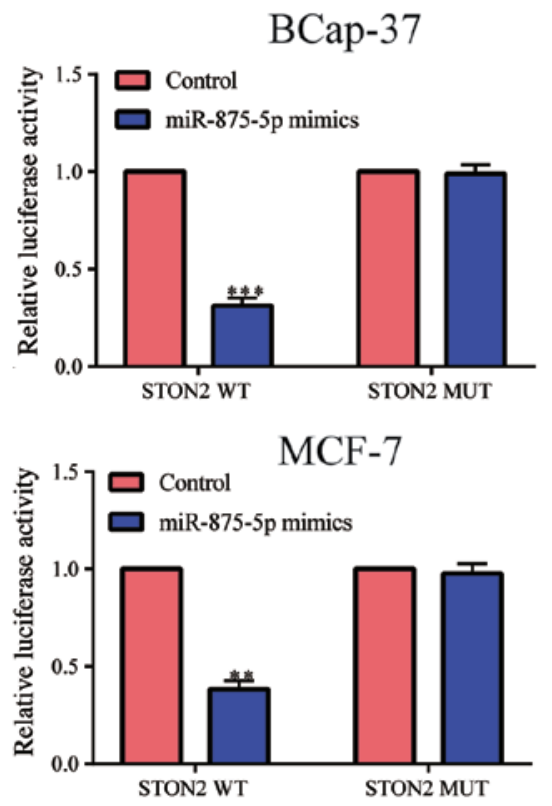

B

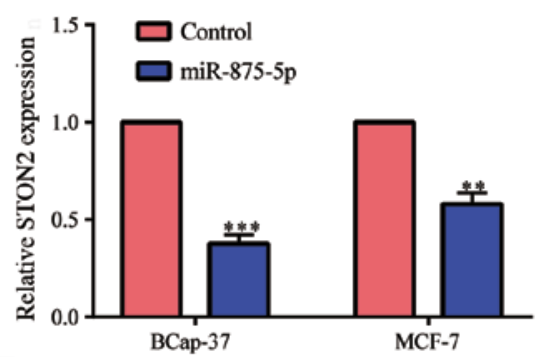

$\mathrm{D}$

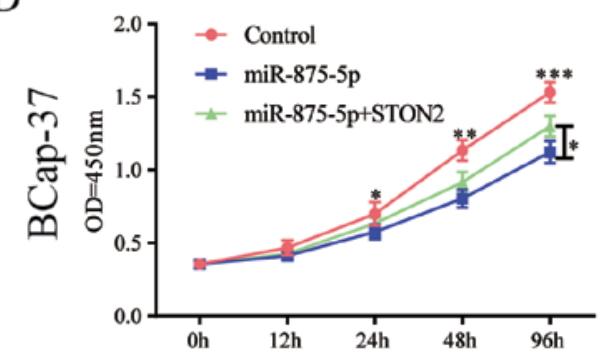

$\mathrm{E}$

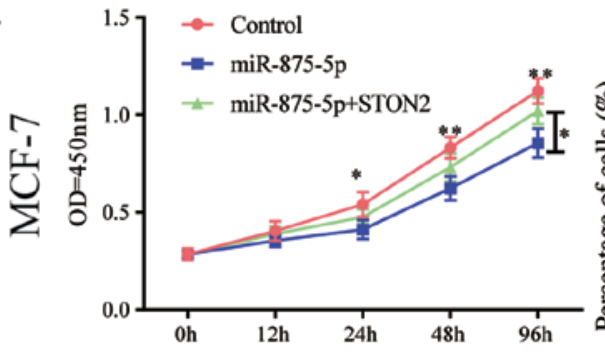

C
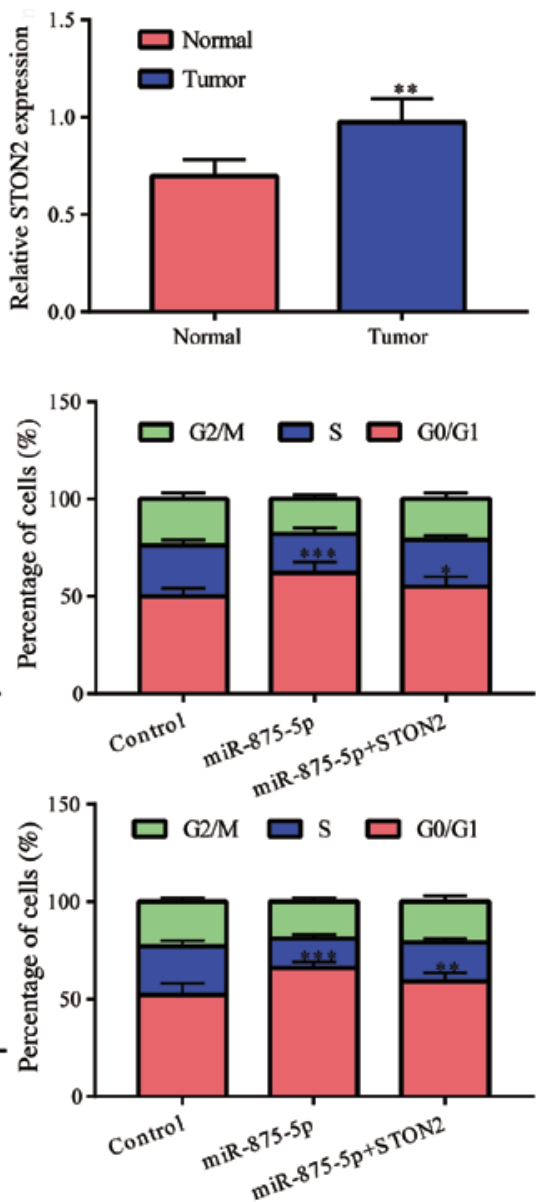

Figure 4. miR-875-5p functions by targeting degradation of Stonin2 (STON2). (A) Dual luciferase reporting assay results indicate that STON2 is a target gene of miR-875-5p. (B) STON2 expression is significantly inhibited after miR-875-5p is overexpressed in MCF-7 and BCap-37 cells. (C) STON2 has a significantly high expression in breast cancer tissues. (D and E) Upregulation of STON2 in MCF-7 and BCap-37 cells can partially abolish the inhibitory effect of miR-875-5p on cell proliferation and cycle. ${ }^{*} \mathrm{P}<0.05,{ }^{* *} \mathrm{P}<0.01$ and ${ }^{* * * *} \mathrm{P}<0.001$.

and MCF-7 cells was partially inhibited by microRNA-875-3p overexpression (Fig. 3D). Moreover, we found that upregulation of microRNA-875-3p in BCap-379 and MCF-7 cells partially inhibited the promoting effect of GACAT1 on the cell cycle (Fig. 3E). Based on the above results, we concluded that GACAT1 may play a role by adsorbing microRNA-875-3p and then regulate its downstream target genes.

microRNA-875-3p functions by targeting degradation of STON2. It was predicted by website that STON2 may be a potential target gene of microRNA-875-3p, and we confirmed their binding relationship by luciferase reporter gene experiment (Fig. 4A). In breast cancer cells, we found that the level of STON2 was conspicuously reduced after overexpressing microRNA-875-3p (Fig. 4B), indicating that STON2 can be degraded by microRNA-875-3p. We examined the level of STON2 in breast cancer tissues and found that it existed at a significant high level in breast cancer (Fig. 4C). To test whether microRNA-875-3p worked by inhibiting the level of STON2, a series of recovery experiments were performed. The results showed that upregulation of STON2 in MCF-7 and BCap-37 cells partially abolished the inhibition of cell proliferation and cycle by microRNA-875-3p (Fig. 4D and E). Based on the above results, we hypothesized that GACAT1 may function by inhibiting the degradation of downstream STON2 by adsorbing microRNA-875-3p.

\section{Discussion}

The competitive endogenous RNA (ceRNAs) hypothesis reveals a novel mode of regulation of RNA-interacting interactions, including messenger RNA (mRNA), pseudogene, long-chain non-coding RNA (lncRNA) and circular RNA (circRNA). By stimulating the same miRNA through microRNA response elements (MREs), the level of miRNA is reduced, thereby reducing or reducing the effect of miRNA on target genes. ceRNA is widely involved in a series of physiological and pathological processes including cell differentiation, proliferation, apoptosis, and growth and invasion of tumor cells $(16,17)$. There have been many studies on the role of ceRNA. Studies have found that lncRNA HOTAIR is conspicuously upregulated in renal cell tumors and acts as an endogenous RNA, which could promote tumor progression by downregulating the level of miR-217. Other studies have shown that lncRNA RMRP also plays a role as a miRNA sponge in lung cancer. By adsorbing miR-206, it upregulates the level of miRNA downstream target genes and plays a role in the development of lung cancer. 
In this study, we found that GACAT1 was highly expressed in breast cancer by qPCR. After staging breast cancer patients and dividing them into metastasis group and non-metastasis group, we further analyzed that GACAT1 level was closely related to breast cancer staging and metastasis, suggesting that GACAT1 could indicate poor prognosis. In vitro, we overexpressed GACAT1 in breast cancer cell lines and found that GACAT1 conspicuously promoted the proliferation and cell cycle of breast cancer cells, suggesting that it may play a role in the development of breast cancer.

To explore whether GACAT1 also functioned as a miRNA sponge, we performed prediction analysis and found that microRNA-875-3p may be a potential target for GACAT1. Previous studies have shown that microRNA-875-3p plays a role as a tumor suppressor gene in a variety of tumors, and its upregulation can often inhibit the proliferation and invasion of tumor cells $(18,19)$. Therefore, we also examined the level of microRNA-875-3p in breast cancer patients, and the results showed that microRNA-875-3p was conspicuously under-expressed in breast cancer. We confirmed the binding relationship between GACAT1 and microRNA-875-3p by luciferase reporter gene assay. Besides, the level of microRNA-875-3p was conspicuously decreased after upregulating GACAT1, indicating that GACAT1 can play the role of ceRNA to downregulate microRNA-875-3p level. To further confirm that GACAT1 functioned through microRNA-875-3p, we simultaneously upregulated GACAT1 and microRNA-875-3p in breast cancer cell lines, and found that microRNA-875-3p partially inhibited the inhibition effect of GACAT1 on cell proliferation and cycle.

In addition, since miRNAs play a role in the targeted degradation of their target genes, we further explored their potential target genes through site prediction and analysis. We found that STON2 may be a potential target gene for microRNA-875-3p. The STON2 gene is a clathrin-related protein that is closely related to the regulation of intracellular complexes $(20,21)$. In ovarian cancer, studies have shown that high level of STON2 can promote tumor invasion and predict a poor prognosis (22). In this study, we verified that microRNA-875-3p could bind to STON2 by dual luciferase reporter gene assay and that STON2 was highly expressed in breast cancer. Through a series of recovery experiments, we found that overexpression of STON2 partially abolished the inhibitory effect of microRNA-875-3p on proliferation and cell cycle of breast cancer cells, indicating that microRNA-875-3p may exert its role through regulating STON2.

Based on the above results, we hypothesized that lncRNA GACAT1 can adsorb microRNA-875-3p to upregulate the level of STON2, thereby promoting the progression of breast cancer.

In conclusion, in this study, we conducted an in-depth study of the role and mechanism of GACAT1 in breast cancer, and demonstrated that GACAT1 could play a vital role in the progression of breast cancer, which also provides theoretical basis for new prevention and treatment for breast cancer.

\section{Acknowledgements}

Not applicable.

\section{Funding}

Not funding was received.

\section{Availability of data and materials}

All data generated or analyzed during this study are included in this published article.

\section{Authors' contributions}

QW and XQ designed the study and performed the experiments, QW and JX collected the data, QR and XL analyzed the data, QW and XQ prepared the manuscript. All authors read and approved the final manuscript.

\section{Ethics approval and consent to participate}

This study was approved by the Ethics Committee of Linyi Cancer Hospital (Linyi, China). Signed informed consents were obtained from the patients and/or guardians.

\section{Patient consent for publication}

Not applicable.

\section{Competing interests}

The authors declare that they have no competing interests.

\section{References}

1. Torre LA, Bray F, Siegel RL, Ferlay J, Lortet-Tieulent J and Jemal A: Global cancer statistics, 2012. CA Cancer J Clin 65: 87-108, 2015.

2. Zhuang $X$ and Wang J: Correlations of MRP1 gene with serum TGF- $\beta 1$ and IL- 8 in breast cancer patients during chemotherapy. J BUON 23: 1302-1308, 2018.

3. Huang NS, Chi YY, Xue JY, Liu MY, Huang S, Mo M, Zhou SL and $\mathrm{Wu}$ J: Long non-coding RNA metastasis associated in lung adenocarcinoma transcript 1 (MALAT1) interacts with estrogen receptor and predicted poor survival in breast cancer. Oncotarget 7: 37957-37965, 2016.

4. Ponting CP, Oliver PL and Reik W: Evolution and functions of long noncoding RNAs. Cell 136: 629-641, 2009.

5. Zhou CX, Wang X, Yang N, Xue SK, Li WC and Xie PP: IncRNA LET function as a tumor suppressor in breast cancer development. Eur Rev Med Pharmacol Sci 22: 6002-6007, 2018.

6. Khan FS, Ali I, Afridi UK, Ishtiaq M and Mehmood R: Epigenetic mechanisms regulating the development of hepatocellular carcinoma and their promise for therapeutics. Hepatol Int 11: 45-53, 2017.

7. Xia T, Liao Q, Jiang X, Shao Y, Xiao B, Xi Y and Guo J: Long noncoding RNA associated-competing endogenous RNAs in gastric cancer. Sci Rep 4: 6088, 2014.

8. Dvinge H, Git A, Gräf S, Salmon-Divon M, Curtis C, Sottoriva A Zhao Y, Hirst M, Armisen J, Miska EA, et al: The shaping and functional consequences of the microRNA landscape in breast cancer. Nature 497: 378-382, 2013.

9. Gibb EA, Vucic EA, Enfield KS, Stewart GL, Lonergan KM, Kennett JY, Becker-Santos DD, MacAulay CE, Lam S, Brown CJ, et al: Human cancer long non-coding RNA transcriptomes. PLoS One 6: e25915, 2011.

10. Sun H, Wang G, Peng Y,Zeng Y,Zhu QN,Li TL, Cai JQ, Zhou HH and Zhu YS: H19 lncRNA mediates 17 $\beta$-estradiol-induced cell proliferation in MCF-7 breast cancer cells. Oncol Rep 33: 3045-3052, 2015. 
11. Xing C, Cai Z, Gong J, Zhou J, Xu J and Guo F: Identification of potential biomarkers involved in gastric cancer through integrated analysis of non-coding RNA associated competing endogenous RNAs network. Clin Lab 64: 1661-1669, 2018.

12. Shi X, Wang X and Hua Y: lncRNA GACAT1 promotes gastric cancer cell growth, invasion and migration by regulating miR-149-mediated of ZBTB2 and SP1. J Cancer 9: 3715-3722, 2018.

13. Lajos R, Braicu C, Jurj A, Chira S, Cojocneanu-Petric R, Pileczki V and Berindan-Neagoe I: A miRNAs profile evolution of triple negative breast cancer cells in the presence of a possible adjuvant therapy and senescence inducer. J BUON 23: 692-705, 2018

14. Takamizawa J, Konishi H, Yanagisawa K, Tomida S, Osada H, Endoh H, Harano T, Yatabe Y, Nagino M, Nimura Y, et al: Reduced expression of the let-7 microRNAs in human lung cancers in association with shortened postoperative survival. Cancer Res 64: 3753-3756, 2004.

15. Cimmino A, Calin GA, Fabbri M, Iorio MV, Ferracin M, Shimizu M, Wojcik SE, Aqeilan RI, Zupo S, Dono M, et al miR-15 and miR-16 induce apoptosis by targeting BCL2. Proc Natl Acad Sci USA 102: 13944-13949, 2005.

16. Salmena L, Poliseno L, Tay Y, Kats L and Pandolfi PP: A ceRNA hypothesis: The Rosetta Stone of a hidden RNA language? Cell 146: 353-358, 2011

17. Rui X, Xu Y, Jiang X, Ye W, Huang Y and Jiang J: Long non-coding RNA C5orf66-AS1 promotes cell proliferation in cervical cancer by targeting miR-637/RING1 axis. Cell Death Dis 9: 1175, 2018.
18. Zhang T, Cai X, Li Q, Xue P, Chen Z, Dong X and Xue Y: Hsa-miR-875-5p exerts tumor suppressor function through downregulation of EGFR in colorectal carcinoma (CRC). Oncotarget 7: 42225-42240, 2016.

19. Wang J, Lu Y, Ding H, Gu T, Gong C, Sun J, Zhang Z, Zhao Y and Ma C: The miR-875-5p inhibits SATB2 to promote the invasion of lung cancer cells. Gene 644: 13-19, 2018.

20. Luan Z, Zhang Y, Lu T, Ruan Y, Zhang H, Yan J, Li L, Sun W, Wang L, Yue W, et al: Positive association of the human STON2 gene with schizophrenia. Neuroreport 22: 288-293, 2011.

21. Kaempf N, Kochlamazashvili G, Puchkov D, Maritzen T, Bajjalieh SM, Kononenko NL and Haucke V: Overlapping functions of stonin 2 and SV2 in sorting of the calcium sensor synaptotagmin 1 to synaptic vesicles. Proc Natl Acad Sci USA 112: 7297-7302, 2015

22. Sun X, Zhang W, Li H, Niu C, Ou Y, Song L and Zhang Y: Stonin 2 overexpression is correlated with unfavorable prognosis and tumor invasion in epithelial ovarian cancer. Int J Mol Sci 18 : $1653,2017$.

(i) $\Theta$ This work is licensed under a Creative Commons Attribution-NonCommercial-NoDerivatives 4.0 International (CC BY-NC-ND 4.0) License. 